\title{
A Novel Computer Assisted Automatic Sleep Scoring System by Employing Artificial Neural Network-A review
}

\author{
Hemu Farooq, Anuj Jain* \\ Department of Electronics and Communication Engineering, Bhagwant University, Ajmer Raj, India
}

Email address:

a1978jain@gmail.com (A. Jain)

${ }^{*}$ Corresponding author

\section{To cite this article:}

Hemu Farooq, Anuj Jain. A Novel Computer Assisted Automatic Sleep Scoring System by Employing Artificial Neural Network-A review. International Journal of Sensors and Sensor Networks. Vol. 5, No. 3, 2017, pp. 43-47. doi: 10.11648/j.ijssn.20170503.12

Received: September 14, 2017; Accepted: September 25, 2017; Published: November 2, 2017

\begin{abstract}
Sleep is an essential element for an individual's well-being and is considered vital for the overall mental and physical heath of a person. Sleep can be considered as a virtual detachment of an individual from his environment. In normal humans, about $30 \%$ of their life-time is spent for sleep. Artificial neural networks (ANNs) or connectionist systems are computing systems inspired by the biological neural networks that constitute animal brains. Sleep scoring is under taken by the examination and visual inspection of polysomnograms (PSG) done by sleep specialist. PSG is specialty test, the conduction of which includes the recording of various physiological signals. The signals obtained are processed using digital processing tools so as to extract information. Soft computing techniques are used to analyze the signals. ANNs are parallel adaptive systems suitable for solving of non-linear problems. Using ANN for automatic sleep scoring is especially promising because of new ANN learning algorithms allowing faster classification without decreasing the performance. Both appropriate preparations of training data as well as selection of the ANN model make it possible to perform effective and correct recognizing of relevant sleep stages. Such an approach is highly topical, taking into consideration the fact that there is no automatic scorer utilizing ANN technology available at present. The high performances observed with systems based onneural networks highlight that these tools may be act new tools in the field of sleep research. In this scenario we are surmised the review regarding the computer assisted automatic scoring of sleep and soft computing technique Artificial Neural Network.
\end{abstract}

Keywords: Sleep Scoring System, Polysomnograms (PSG), Artificial Neural Network (ANN)

\section{Introduction}

Sleep is an essential element for an individual's well-being and is considered vital for the overall mental and physical heath of a person. Sleep is a state in which the physical and mental functions of a human being are in a state of a pause, as well as various external stimulations do not affect the individuals which is in the resting state [1]. Thus sleep can be considered as a virtual detachment of an individual from his environment. In normal humans, about $30 \%$ of their life-time is spent for sleep [2]. The concept of sleep is considered to be highly mysterious and a topic of discussion and research interest since ages and $t$ has fascinated the researchers all over the world [3]. Prolonged sleep disorders or a continuing sleep insufficiency invites various disease of heart, kidney, etc and increases the risk of contracting long term passive diseases like diabetes, high blood pressure as well as stroke in extreme cases. These are a source of discomfort and distress to the individual experiencing them such as insomnia, sleep walking, narcolepsy and nocturnal breathing disorders and hence, they need to be treated [4].

Sleep scoring is an important aspect in the field of sleeps research as well as sleep medicine. Classically, for years together, the sleep scoring has been under taken by the examination and visual inspection of polysomnograms (PSG) done by a sleep specialist [5]. Here, PSG is specialty test, the conduction of which includes the recording of various physiological signals like Electroocularogram (EOG): the records of eye movements both for left and right eyes, Electroencephalograph (EEG):a measure of well-known 
brain waves generated by cortex and other integrative processing mechanism, Respiration, Electromyogram (EMG):the record of electrical activity that emanates from active muscles and pulse Oximetry [6]. All of these signals when recorded for a short period of time called epoch and analysed indicate different stages of sleep. The Rechtschaffen and Kales manual for normal sleep classifies the epochs and scores them as waking, Rapid Eye Movement (REM), NonRapid Eye Movement (NREM) as the basic three stages of sleep. Further, there is a classification provided NREM four stages of sleep. These stages are scored according to the various signals recorded in that particular epoch [7].

\subsection{Sleep Scoring System}

Sleep stage scoring is a rule-based neurophysiological characterization requiring an understanding of the basic mechanisms underlying the generation of cephalic electric potentials coupled with eye movements and muscle signals. Signals of interest are generated from the brain (ie, cortex and deeper structures), facial muscles as noted by the submental EMG, and eye movement as detected by the EOG [8]. Interference with the signals of interest is encountered through many mechanisms, including physiological attenuation of the cerebral electric potentials by scalp muscle and bone, intrusion into the signal by slow cyclic respiration, movement, ECG signal, external electric fields, and impaired contacts between the recording electrodes and the skin surface. Discriminating true signal from artifact can be one of the most challenging aspects of scoring sleep stages [9].

\subsection{Artificial Neural Network (ANN)}

Artificial neural network is a widely employed soft computing technique. They have been used for a variety of purposes like pattern recognition, analysis, classification, along with functional monitoring in fields concerned with signals and systems. Its robustness and flexibility has made it popular for computing in a wide array of fields [10]. The optimum balancing of equation of designing ease, time of execution and the level of accuracy has made it a highly used classifier when large amount of computing is required [11].

The fundamental design and modeling of a neural networks is inspired from the biological neural systems. Usually in the neural networks designed for such computations are much simpler structures and possess the abilities of learning and reacting [12]. The primary features of neural networks which are adopted for computational purpose are its ability to adapt as well as its characteristic of non-algorithm and parallel-distributed memory [13].

A standard feed forward neural network consists of three layers of operation viz. input layer, hidden layer, output layer. There can be one or more hidden layers which perform an array of different functions. The hidden layers are made up of cells which undertake the function of summing up the output which is a product of preceding layer [14]. This summing is done after multiplying it by weight vector through neurons, Hence, each cell or anode gives a resultant output with the given input according to a non-linear transfer function which is known as the activation function [15].

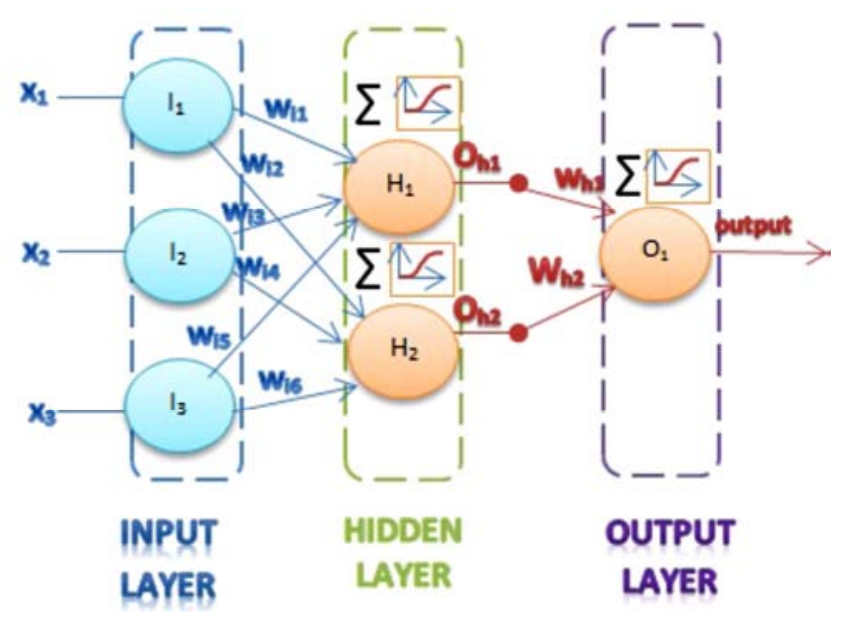

Figure 1. A standard feed forward neural network.

The determination of number of hidden layers is the critical task when adopting and designing the artificial neural network. It is unknown at the starting of the process unlike the input and the output layer which have a precise definition from the beginning. Hence, the input is fed and the output is observed by the neural network [16]. With numerous such iterations, and by employing the trial and error method, the number of hidden layers and their activation functions are calculated gradually by the artificial neural network. This process is called the training phase of the artificial neural network which may be time consuming [17]. The artificial neural network must be trained to function as per requirement of the computation at hand. Usually an artificial neural network is expected to have a number of hidden nodes because the one possessing a smaller number of nodes comes with the constrained capability of distinguishing between complex patterns [18].

\subsection{Artificial Neural Network Technology}

Artificial neural networks are computational tools composed of a large number of highly inter connected elementary processors (also named 'cells' or 'neurons'). Information is stored in each processor as intensities (also named 'weights') of its connections [19]. The basic idea of connectionism is that global coherent behavior can emerge from such organization. Though a wide variety of artificial neural network models exist, each can be completely defined by three characteristics:

\subsubsection{The Topology of the Network}

This characteristic relates to the network organization. Data are generally presented to the network through a set of input cells. After a processing phase, the result is available through a set of cells referred toas output cells [20]. These cells interact with the external world but other cells with no direct interaction with the external world can exist (they are referred to as hidden cells). Each connection is characterized by its intensity (weight) and by the direction of propagation 
of the information. An individual network is characterized by the numbers of cells and the connections between the cells. The most employed model is the multilayer perceptron wherein cells are set in successive layers [21]. The input layer is separated from the output layer by one (or more) hidden layer(s). Each layer is generally fully connected to its adjacent layer, information being processed from the input layer to the output layer [22].

\subsubsection{The Transfer Function of Each Cell}

Each cell sums up the weighted inputs it receives from other cells and generates an output value (also referred to as the state of the cell) using a transfer function. This transfer function is usually a sigmoid ora binary function with the output value generally evolving between two limits ( -1 and +1 or 0 and +1 ) [23].

\subsubsection{The Learning Rule}

To process data properly, the intensity of each connection is adjusted during a training phase which orients the global behavior of the network toward the one expected by the operator. The learning rules applied during the training phase can be divided into two main classes; supervised and unsupervised learning rules [24]. The unsupervised learning procedures strive to catch regularities in the input data. The supervised learning procedures require external intervention (performed by a 'teacher') to guide the training phase. The principle of supervised learning is to provide the network with a set of reference 'patterns' with which adequate weights are computed. With the multilayer perceptron, the most frequently employed learning rule is the back propagation algorithm described by Rumelhart et al. (1986) [25]. This algorithm is based on the presentation by a teacher, to the network, of vectors (an input vector and the expected output vector). Information from the input layeris thus propagated down through the network to the output layer. After propagation, the quadratic error computed from the observed and expected outputs isused to modify the value of each weight in the network [26]. When the learning phase is ended, the network is supposed to associate any input vector with an appropriate output vector. Further details concerning artificial neural network computation can be found in Leuthausser, Freeman and Skapura [27], [28].

\section{Historic Overview}

Krausman et al. used method and an apparatus which were disclosed for scoring the quality of sleep experienced for a specific period [29]. This sleep monitoring apparatus includes a motion server that quantifies the temporal variation in the subject's motion, an analog to digital converter to sample the sensor data, a microprocessor with embedded programmable memory to store control and processing firmware. The firmware for this apparatus directs the microprocessor to a sample space sensor output directs the microprocessor to analyze temporarily stored data to compute a sleep score, controls the operation of display means. A high score indicates restful sleep (very low movements) while a low score indicates excessive restlessness during monitored sleep period [30].

Park et al. proposed an automated method for sleep stage scoring using hybrid rule and case based reasoning [31]. The method comprised of signal processing unit, rule based scoring unit and case based scoring unit. Authors applied this methodology to three recordings of normal sleep and three recordings of obstructive sleep apnea (OSA). Average agreement rate in normal recording was $87.5 \%$ and case based scoring showed agreement rate by $5.6 \%$. This showed several advantages in sleep scoring: high performance on sleep disorder recordings and learning ability. Silber et al. have discussed the work of AASM visual scoring task force behind the new rules [32].

Reliability studies of Rechtschaffen and Kales ( $\mathrm{R}$ and $\mathrm{K}$ ) scoring were reviewed; reliability was low for stage 1 and moderate for slow wave sleep which was also supported by the findings of Moser et al. [34], [35], [33]. All night polysomnographic recording were scored visually according to $\mathrm{R}$ and $\mathrm{K}$ rules and AASM rules by experienced sleep scorers. Healthy subjects (38 females and 34 males) aged between 21 and 86 years were taken. While sleep latency, total sleep time, sleep efficiency were not affected by classification standard, time spent in sleep stage $1(\mathrm{~S} 1 / \mathrm{N} 1)$, stage $2(\mathrm{~S} 2 / \mathrm{N} 2)$ and slow wave sleep $(\mathrm{S} 3+\mathrm{S} 4 / \mathrm{N} 3)$ differed significantly between $\mathrm{R}$ and $\mathrm{K}$ and AASM classification.

Ming Ming Liu et al. identified that EMG signals for dynamically contracting muscle have never been used to predict experimentally known muscle force across subjects [36]. Authors used an Artificial Neural Network (ANN) approach to first derive an EMG-force relationship from a subset of experimentally determined EMGs and muscle forces; second, used this relationship to predict individual muscle forces for different contractile conditions and in subjects whose EMG and force data were not used in the derivation of EMG-force relationship; and third validated the predicted muscle forces against the known forces recorded in vivo. Considering the conceptual differences in the tasks investigated (e.g. slow walking vs. trotting), the intra-subject results obtained were superior to those published previously. The inter-subject results typically gave cross-correlation coefficients between actual and predicted forces of $>0.90$ and root mean square errors of $<15 \%$.

Subasi et al. have used an accurate and computational efficient means of classifying EMG signal patterns. In this study, authors used feed forward error back propagation artificial neural network (FFBANN) and wavelet neural network (WNN) based classifiers for comprising their accuracy in classification of EMG signals. The success rate for WNN technique was $90.7 \%$ and for FFEBANN technique $88 \%$ [37].

Wang et al. determined muscle activations from EMG signals by using neural network. The feed-forward neural network model of muscle activation is composed of four layers. Thus, an adjusted back-propagation algorithm was developed. Once muscle activations were obtained and hence used to estimate muscle force. The result obtained showed 
that this neural network model can be used to represent relationship between EMG signal and joint movements [38].

\section{Uses and Application of Artificial Neural network (ANN)}

\subsection{Uses}

1. In learning algorithm, as any algorithm will work well with the correct hyper parameters for training on a particular data set. However, selecting and tuning an algorithm for training on unseen data requires significant experimentation

2. If the model, cost function and learning algorithm are selected appropriately, the resulting ANN can become robust.

3. It is used as function approximation, or regression analysisincluding time series prediction, fitness approximation and modeling.

4. It can be used as a classifier which involves the pattern and sequence recognition.

5. ANN has found capabilities in data processing which includes filtering, clustering, blind source separation and compression.

\subsection{Applications}

The system which estimates sleep stages of human beitttng through a multi-layer neural network (NN) that simultaneously employs EEG, EMG and EOG. The data were recorded through polisomnography device. These collective variant data were first grouped by an expert physician and the software of polisomnography, and then used for training and testing the proposed Artificial Neural Network (ANN). A good scoring was attained through the trained ANN, so it may be put into use in clinics where lacks of specialist physicians.

\subsection{Other Application}

Because of their ability to reproduce and model nonlinear processes, ANNs have found uncountable applications to a wide range of disciplines.

1. Application areas include system identification and control (vehicle control, trajectory prediction, process control, natural resources management), quantum chemistry, game playing and decision making (chess, poker), pattern recognition (radar systems, face identification, signal classification, object recognition and more), sequence recognition (gesture, speech, handwritten text recognition), medical diagnosis, finance (e.g. automated trading systems), data mining, visualization, machine translation, social network filtering and e-mail spam filtering.

2. ANNs have been used to diagnose cancers, including lung cancer, prostate cancer, colorectal cancer and to distinguish highly invasive cancer cell lines from less invasive lines using only cell shape information.
3. ANNs have been used for building black-box models in geosciences: hydrology, ocean modeling and coastal engineering, and geomorphology are just few examples of this kind.

\section{Conclusion}

The research would result in an effective system for computer assisted automatic sleep scoring, which would employ artificial neural network for its functioning. The data collected for the analysis would be the set of the signals obtained from the patient's body. These electrical signals can be collected with the help of polysomnograph (PSG) from a sleep laboratory or clinic in the vicinity of researcher and to which the researcher has accessibility. Once the data collected, it would be applied to the classifier that is Artificial neural network for its classification. Although, the artificial neural network is a highly research topic, the utilization of the same for biomedical purpose of sleep scoring is novel when employed with electrical signals acquired from body. The novel technique of sleep scoring could be of greater significance in various applications for neurological clinics, and particularly helping the neurologist in their purpose of the wake and sleep correlates as well as in the diagnosis of certain sleep disorders.

\section{References}

[1] ZT. Yeh, RP. Chiang, SC. Kang, CH. Chiang "Development of the Insomnia Screening Scale based on ICSD-II.", Int J Psychiatry Clin Pract, 16(4), pp. 259-267, Oct. 2012.

[2] HJ. Park, JS. Oh, DU. Jeong, KS. Park "Automated Sleep Stage ScoringUsing Hybrid Rule- and Case-Based Reasoning.", Computers andBiomedical Research, 33, pp. 330-349, 2000.

[3] David T. Krausman, Bel Air, Richard P. Allen, "Sleep Scoring apparatus and method. Google Patents," 12 April 2005.

[4] Park, H. J., Oh, J. S., Jeong, D. U., Park, K. S., “Automated Sleep Stage Scoring using hybrid rule and case based reasoning", Computers and Biomedical Research, 2000, 33(5), pp.330-349.

[5] Vani Rao, M. D., Alyssa Bergey, B. A., Hugh Hill, M. D., David Efron, M. D., and Una McCann, M. D. Clinical Research Reports, Sleep Disturbance After Mild Traumatic Brain Injury: Indicator of Injury?he Journal of Neuropsychiatry and Clinical Neurosciences. 23: 2; 201-205, 2011.

[6] Penzel, T., Conradt, R., Computer based sleep recording and analysis. Sleep Med. Rev. 4:131-138, 2000.

[7] Moser, D., Anderer, P., Gruber, G., Parapaties, S., Loretz, E., Boeck, M., Danker-Hopfe, H., "Sleep Classification according to AASM and Rechtschaffen and Kales: effects on sleep scoring parameters", Sleep, 2009, 32(2), pp.139-149.

[8] Silber, M. H., Ancoli-Isreal, S., Bonnet, M. H., Chokroverty, S., Grigg-Damberger, M. M., Hirshkowitz, M., Penzel, T., "The visual Scoring of Sleep in Adults", J Clin Sleep Med, 2007, 3(2), pp.121-131. 
[9] Marzec, M. L., Malow, B. A., "Approaches to staging sleep in polysomnographic studies with epileptic activity", Sleep Med. Sep 2003; 4(5): 409-17.

[10] Sonali, B., Maind, Priyanka Wankar, "Research paper on basic of Artificial Neural Network", International journal on recent and innovation trends in computing and communication, Jan 2014; 2321-8169.

[11] Ming Ming Liu, Walter Herzog Hans. H. C. M. Savelberg., "Dynamic muscle force prediction from EMG: an artificial neural network approach", Journal of Electromyography and Kinesiology, 1999, 9(6), pp.391-400.

[12] Shimada, T., Shiina, T., Saito, Y., "Detection of characteristic waves of Sleep EEG by neural network analysis", IEEE Transactions on Biomedical Engineering, 2000, 47(3), pp. 369-379.

[13] Tagluk, M. E., Sezgin, N., and Akin, M., "Estimation of Sleep Stages by an Artificial Neural Network employing EEG, EMG, EOG”, Journal of Medical System, 2010, 34(4), pp. 717-725.

[14] Mousmita Sarma, Kandarpa Kumar Sarma, "Fundamentals consideration of ANN", Phoneme-Based speech segmentation using hybrid soft computing frame work, April 2014; 47-75.

[15] Wang, L., and Buchannan, T. S., "Prediction of Joint Movements using a Neural Network model of Muscle Activation from EMG Signals", IEEE Transaction on Neural System and Rehabilitation Engineering, 2002, 10(1), pp. 3037.

[16] Kulkarni, P., Ade R., "Incremental learning from unbalanced data with concept drift and missing features: a review", International journal of data Min knowledge management process, 2014; 15-29.

[17] Mehmet Akin, Muhammed B., Kurt, "Estimating vigilance level by using EEG and EMG signals", Neural computing and applicatons, 2008, pp. 227-236.

[18] Subasi, A., Yilmaz, M., and Ozcalik, H. R., "Classification of EMG Signals using Wavelet Neural Network", Journal of Neuroscience Methods, 2006, 156(1), pp. 360-367.

[19] Claude Roberta, Christian Guilpinb, Ayne Linuogea, "Review of Neural Network Applications in sleep research", Journal of Neuroscience Method, 1998, pp. 187-193.

[20] Enrique Dominguez Merino, Jose Munoz-Perez, "An efficient Neural Network Algorithm for p-medium problem", Advances in Artificial Intelligence, 2002, pp. 460-469.

[21] Gevins A. L., Morgan N. H., "Applications of neural networks signal processing in brain research", IEEE Transcations on Acoustics, speech and signal processing, 1998; 36(7): 11521161.

[22] Grozinger M., Freisleben B., Roschke, "Comparison of back propagation neural network and a non parametric discriminant analysis in the evaluation of sleep EEG data", Pro of the world of congress on NN, 1994; 1: 462-466.
[23] Roschke J., Kloppel B., “Automatic recognition of REM sleep by ANN”, Sleep Research, 1995; 4: 86-91.

[24] Hasan, J., "Automatic analysis of sleep recordings: A critical review", Annals of clinical research, 1985; 17: 280-287.

[25] Hertz J., Kroch A., Palmer R., "Introduction to the theory of neural computation", Wesley publication company, 1991; 326.

[26] Kemsley D. H., Martinez T. R., Campbell D. M., "A survey of neural network research and fielded applications", International journal of neural networks, 1991; 2: 123-132.

[27] Miler A. S., Blott B. H., Hames T. K., "Review of neural network applications in medical imaging and signal processing" Med and Bio Eng and computation 1992; 30: 449464.

[28] Principe J. C., Tome A. M. P., "Performance and training strategies in feed forward NN: an application to sleep scoring, Proceeding of the IJCNN' $89 ; 1: 341-346$.

[29] Eric A. Nofzinger, Jeffrey J., Damian F., "Apparatus and method for modulating sleep. Google Patents", 2015.

[30] Conor Heneghan, Conor Hanley, Niall Fox, "Apparatus, System and method of monitoring physiological signs. Google Patents", 2013.

[31] Park H. J., J. S. Oh, D. U. Jeong, K. S. Park “Automated Sleep Stage ScoringUsing Hybrid Rule- and Case-Based Reasoning.", Computers andBiomedical Research, 33, pp. $330-349,2000$.

[32] Grigg-Damberger, M. M., "The AASM Scoring Manual four years later”, J clin Sleep Med 2012; 8(3): 323-332.

[33] Luana Novelli, Raffaele Ferri, Oliviero Bruni, "Sleep classification according to AASM and $\mathrm{R}$ and $\mathrm{K}$ : effects on sleep scoring parameters of adults", Journal of sleep research, 2010, pp. 238-247.

[34] Doris Moser, Peter Anderer, Georg Gruber, Georg Dorffner, "Sleep classification according to AASM and Rechtschaffen and Kales", Sleep, March 2009, 32. 2. 139.

[35] Silvia Miano, Maria Chaira Paolino, Rosa Castaldo, Maria Piavilla", Visual Scoring of sleep: A comparison between R and K criteria and AASM criteria", Clinical Neurophysiology, 2010, pp. 39-42.

[36] Yanfenz H., Jacek M. Z., W. Karwowski, William S. M., “A hybrid Neuro-Fuzzy approach for spinal force evaluation", Proceedings of first Internal conference on Advances in Natural Computation, 2005, pp. 1216-1225.

[37] Abdulhamit Subasi, Mustafa Yimaz, Hasan Riza Ozcalik, "Classification of EMG Signals using Wavelet Neural Network", Journal of Neuroscience Methods, 2006, pp. 360367.

[38] Lin Wang, T. S. Buchanan, "Prediction of joint moments using NN model of muscle activation from EMG signals", IEEE Transaction on Neural system and Rehabilitation Engineering, 2002, pp. 30-37. 